J3eA, Journal sur l'enseignement des sciences et technologies de l'information et des systèmes, Volume 2, Hors-Série 2, 9 (2003)

DOI : http://dx.doi.org/10.1051/bib-j3ea:2003609

(C) EDP Sciences, 2003

Évolution de l'intégration en électronique de puissance

M. Breil et F. Morancho

LAAS-CNRS

7 avenue du colonel Roche

F-31077 Toulouse Cedex 4, France

breilelaas.fr, morancho@laas.fr 


\section{Évolution de l'intégration en électronique de puissance}

\author{
Marie Breil \\ LAAS-CNRS \\ 7, avenue du Colonel Roche \\ 31077 Toulouse Cedex 4
}

\author{
Frédéric Morancho \\ LAAS-CNRS \\ 7, avenue du Colonel Roche \\ 31077 Toulouse Cedex 4
}

E-mail : breil@laas.fr, morancho@laas.fr

\section{Résumé}

Dans ce papier, l'évolution de l'intégration en électronique de puissance est présentée. Les différents types d'intégration sont abordés (hybride et monolithique). Une attention plus particulière est portée sur les deux grandes familles de l'intégration monolithique que sont l'intégration de puissance Smart Power et l'intégration fonctionnelle. Il apparaît clairement que l'intégration en électronique de puissance évolue vers une intégration de systèmes de puissance, comprenant les composants de conversion de l'énergie, des composants passifs, des éléments de commande et de protection et des éléments concernant l'environnement $d u$ composant.

\section{Introduction}

En électronique de puissance, les fonctions sont principalement liées aux opérations de contrôle et de conversion de l'énergie électrique. Ainsi, les semiconducteurs de puissance sont principalement destinés à des fonctions d'interrupteurs dans la mise en œuvre des convertisseurs d'énergie (redresseur, hâcheur, onduleur, etc...). Toutefois, des fonctions spécifiques de protection des équipements électriques mettent également en jeu des semi-conducteurs de puissance.

Les premiers composants de puissance (diodes, transistors bipolaires, thyristors) permettant de contrôler des tensions et des courants élevés furent commercialisés dans les années 50 et, depuis, les composants semiconducteurs se sont progressivement substitués aux solutions électromécaniques pour la réalisation des convertisseurs d'énergie. Dans les années 70, les structures de type MOS (Métal-Oxyde-Semiconducteur), caractérisées par une impédance d'entrée élevée, ont permis de s'affranchir des commandes en courant des dispositifs de puissance purement bipolaires. Les premiers transistors MOS de puissance ont donc vu le jour, préfigurant les nombreux composants de puissance basés sur l'association des technologies MOS et bipolaires. Ainsi, au cours des années 80, un pas technologique fut franchi avec l'IGBT (Insulated Gate Bipolar Transistor) qui est devenu le composant de puissance le plus utilisé pour les applications de moyenne puissance. Depuis une quinzaine d'années, l'intégration en électronique de puissance s'est développée, tirée par des marchés spécifiques comme l'automobile, l'éclairage ou l'électroménager. Suivant les niveaux de puissance et les contraintes à supporter, les solutions d'intégration sont hybrides ou monolithiques. Dans une première partie, nous évoquerons l'intégration hybride ; la deuxième partie sera consacrée à l'intégration monolithique et ses deux grandes familles que sont l'intégration "Smart Power" et l'intégration fonctionnelle.

\section{L'intégration hybride}

Ce mode d'intégration est adapté aux applications fonctionnant dans des gammes en puissance supérieures à celles couvertes par l'intégration monolithique, typiquement pour des courants supérieurs à $30 \mathrm{~A}$ et des tensions se situant dans la fourchette $600 \mathrm{~V} / 1200 \mathrm{~V}$. Dans ces gammes de puissance, il est avant tout nécessaire de recourir à un mode d'intégration conduisant à une bonne évacuation de la chaleur et à une isolation galvanique parfaite entre les différents éléments. En outre, les avantages de ce mode d'intégration sont nombreux : ils concernent principalement la réduction des coûts, la réduction des dimensions, un montage plus simple, la réduction des inductances et la réduction de la résistance thermique. L'intégration hybride, qui se situe à mi-chemin entre l'intégration monolithique et le discret, permet d'associer sur un même substrat les divers composants mis en jeu dans la réalisation d'une fonction de puissance "intelligente" grâce à l'utilisation simultanée de maténiaux adéquats et de méthodes d'assemblages adaptées. Toutefois, il faut distinguer les modules de puissance intelligents, dont l'appellation anglo-saxonne est IPM (Intelligent Power Module) et les modules standards, en fonction des gammes de puissance visées.

\subsection{Modules de puissance intelligents (IPM ou ASIPM)}

Ils sont utilisés pour des tensions supérieures à $600 \mathrm{~V}$ et des courants compris entre $4 \mathrm{~A}$ et $100 \mathrm{~A}$. L'idée de modules de puissance intelligents est née au début des années $90 \mathrm{du}$ besoin toujours croissant d'associer le maximum de fonctions sur un minimum de surface. Ces modules sont réalisés en intégrant dans un "boîtier puce" des composants de puissance (principalement des IGBT) et les diodes de roue libre associées, avec leurs circuits de commande et de protection. Pour combler les lacunes des IPM pour des applications qui nécessitent des circuits de commandes complexes très compacts, les ASIPM (Fig. 1) ont été développés pour augmenter encore d'un cran la fonctionnalité du dispositif intégré.

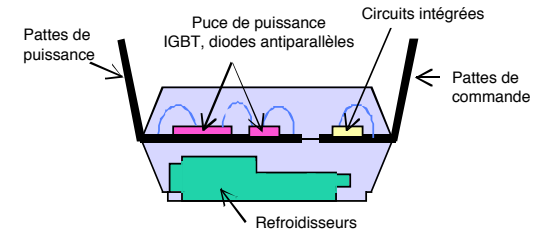

Figure 1. Coupe d'un ASIPM [1].

Les moyens mis en œuvre permettent d'intégrer, avec les composants de puissance, des circuits intégrés de puissance (HVIC, High Voltage Integrated Circuits) en plus des ASIC déjà utilisés dans les IPM. 


\subsection{Modules standard de puissance}

Ils concernent les applications de forte puissance (de 100 à $2000 \mathrm{~A}$ et de $1200 \mathrm{~V}$ à $3500 \mathrm{~V})$. Dans ce cas, l'intégration se réduit à l'association en parallèle d'IGBT et de leurs diodes de roue libre. Ces modules utilisent des méthodes d'assemblage classiques ; la puce repose sur un substrat et les fils de contact en aluminium sont montés sur la surface de la puce par soudure à ultrason. Cependant, il faut noter que la fiabilité de ces modules est faible car ces derniers subissent d'importantes contraintes thermiques, qui ont pour conséquence d'endommager, par effet de dilatation, les différentes couches qui servent de support à la puce. De plus, les connexions de grille et de cathode qui sont liées aux contacts d'aluminium à la surface de la puce sont particulièrement vulnérables aux échauffements. L'amélioration de ces dispositifs viendra donc principalement d'un agenœment optimisé de matériaux mieux adaptés aux cycles thermiques que subissent les composants aux cours des cycles de fonctionnement.

\section{L'intégration monolithique en électro- nique de puissance}

Bien que les performances à optimiser pour les composants de puissance soient différentes de celles des circuits intégrés, l'évolution des composants de puissance au cours des vingt dernières années est étroitement liée aux progrès des technologies microélectroniques réalisés dans le domaine des circuits intégrés. En effet, l'essor important des circuits intégrés du traitement du signal et de l'information s'est accompagné d'un important effort de recherche qui a conduit au développement de nouveaux procédés technologiques et à la réduction des dimensions.

La synergie entre les domaines de la puissance et du traitement du signal a été déclenchée par l'introduction des technologies MOS dans les dispositifs de puissance. Les performances en terme de résistance à l'état passant des structures MOS verticales de puissance (VDMOS) ont été nettement améliorées par la réduction des dimensions qui a permis d'augmenter le nombre de cellules par unité de surface. L'IGBT, qui est actudlement l'un des dispositifs les plus utilisés en électronique de puissance, est une retombée directe de ces travaux. Les dispositifs "Smart Power" mixant des dispositifs de puissance à commande MOS et des circuits de traitement du signal illustrent parfaitement cette tendance [2,3].

La réalisation technologique de ces nouvelles fonctions de puissance intégrées peut se traiter de deux façons, soit en privilégiant la fonctionnalité aux dépens des éléments de puissance, soit en favorisant l'optimisation de la partie puissance. Les dispositifs Smart Power et HVIC correspondent davantage à la première approche et sont réalisés à partir de filières technologiques de type circuits intégrés (CMOS ou BiCMOS). Les dispositifs de puissance discrets intelligents et l'intégration fonctionnelle procèdent de la deuxième approche, et sont basés sur des technologies de composants de puissance.

\subsection{Les circuits intégrés "Smart Power"}

Les progrès technologiques ont permis d'intégrer sur une même puce les composants de puissance et les circuits analogiques comportant une logique complexe et même parfois associant des mémoires. Ainsi, les premiers circuits intégrés de puissance pour des applications faible tension sont appanus dès 1985, quinze ans après les débuts de l'intégration des composants de signal, sous deux formes, les circuits Smart Power et les circuits HVIC [4]. La différence entre les technologies utilisées pour la conception de ces deux types de circuits vient essentiellement de l'agencement de l'élément de puissance et des gammes en courant et en tension traitées : en Smart Power, le composant de puissance, généralement seul, peut être horizontal ou vertical (DMOS), alors que pour les HVIC, les composants de puissance sont latéraux et optimisés pour supporter des tensions importantes à l'état bloqué (LDMOS). Les HVIC sont des circuits multi-sorties pouvant supporter des tensions jusqu'à quelques centaines de Volts mais présentant des densités de courant très faibles inhérentes aux composants latéraux utilisés. Au contraire, les composants Smart Power, plus performants en terme de densités en courant, peuvent transiter des courants de plusieurs Ampères avec néanmoins des tensions blocables inférieures à celles des HVIC. Dans ce type de fonctions, la partie logique et analogique du circuit réalisé en technologie NMOS, CMOS ou bipolaire doit être parfaitement isolée du composant de puissance subissant des contraintes importantes en terme de courant, tension, $\mathrm{di} / \mathrm{dt}$ et $\mathrm{dv} / \mathrm{dt}$.

Les premières générations de dispositifs intégrés de puissance furent réalisées avec une technologie utilisée pour la conception des composants de puissance, ne permettant pas, par conséquent, d'atteindre des densités d'intégration très grandes. Les nouvelles générations de composants Smart Power (Fig. 2), SmartMOS (Motorola) ou BCD (ST Microelectronics) sont conçues à partir de technologies VLSI qui doivent être en mesure de permettre la conception de composants de puissance capables de supporter des tenues en tension de l'ordre de 100 Volts en utilisant des techniques d'isolation développées ces dernières années.

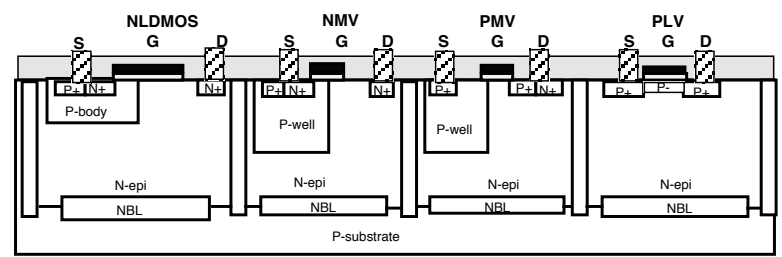

Figure 2. Exemple d'intégration de composants "Smart Power".

Néanmoins, bien que la technologie Smart Power ait apporté au composant de puissance une "autonomie intelligente" qu'il ne possédait pas jusqu'alors, elle trouve ses limites dans les méthodes d'isolation électrique utilisées entre les éléments de puissance et l'électronique de commande. A l'heure actudle, les composants Smart Power ne peuvent donc êtres utilisés que dans le domaine des faibles puissances, comme dans l'automobile et les télécommunications. L'étude des techniques d'isolation entre la partie basse tension et le (ou les) élément(s) de puissance a constitué l'un des chantiers les plus importants de cette famille de composants. L'auto-isolation, l'isolation par jonction et l'isolation par diélectrique sont les principales solutions utilisées aujourd'hui. Toutefois l'isolation par diélectrique reste encore coûteuse et réservée, malgré son efficacité, aux applications nécessitant une très bonne isolation. Ces différentes techniques ne permettent pas d'assurer des isolations supénieures à quelques centaines de Volts. Ce mode d'association s'adresse donc à des applications de faible puissance et sans contraintes fortes d'isolation galvanique. Les grands domaines d'application basés sur des sources autonomes sont aujourd'hui l'électronique automobile et les télécommunications.

Ces technologies permettent d'intégrer des circuits digitaux complexes (DSP) et des microcontrôleurs. Alors que dans les premiers circuits Smart Power la surface du composant de puissance était souvent supénieure à celle de 
la partie "cirait intégré", la tendance est inversée dans les nouveaux circuits intégrés de puissance qui se caractérisent par une intégration poussée de nouvelles fonctionnalités. Cette tendance est accompagnée par une réduction des règles de dessin et des filières technologiques évoluées. La Figure 3 présente un schéma bloc de ce type de circuit que l'on peut qualifier de "nouveau Smart Power". On peut distinguer trois parties : les circuits d'interface, les circuits de contrôle et de traitement du signal et l'élément de puissance. Au niveau des circuits d'interface, la tendance consiste à remplacer les circuits bipolaires par des circuits BiCMOS présentant des performances plus intéressantes. Les circuits de traitement du signal correspondent à des fonctions CMOS présentant une faible puissance de consommation et une forte densité d'intégration. Les dispositifs de puissance sont généralement basés sur des technologies DMOS, permettant de réaliser des structures latérales (LDMOS), ou verticales (VDMOS). Dans la perspective d'augmenter la fonctionnalité, des mémoires peuvent également être intégrées. Les technologies BCD (bipolaire, CMOS et DMOS) permettent une flexibilité importante pour réaliser les différents types de circuits précités.

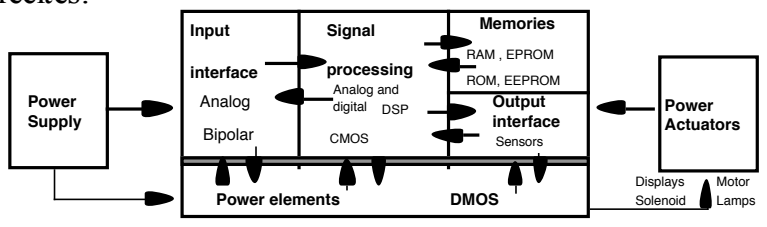

Figure 3. Schéma bloc d'un circuit DSP.

Notons que l'industrie des composants de puissance bénéficie depuis des avancées technologiques propres à la réalisation des mémoires et des microprocesseurs avec cependant un certain retard (Fig. 4). En 2000, ce retard n'était plus que d'une génération et les longueurs des canaux des transistors MOS atteignaient $0,35 \mu \mathrm{m}$ pour les circuits intégrés de puissance contre $0,18 \mu \mathrm{m}$ en électronique VLSI (Very Large Scale Integration).

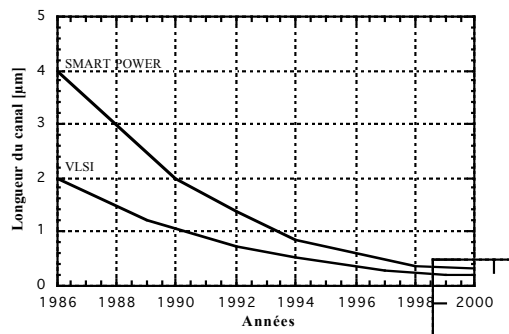

Figure 4. Com paraison entre l'évo lution des techn ologies Smart Power et VLSI.

\subsection{Intégration fonctionnelle}

Il existe un grand nombre d'applications, alimentées à partir du réseau de distribution électrique (conversion d'énergie et protection dans les secteurs industriel et également domestique) pouvant bénéficier des avantages apportés par l'intégration monolithique. Ainsi, l'intégration fonctionnelle a permis de franchir une nouvelle étape grâce au développement de nouvelles fonctions de puissance intégrées, pouvant être utilisées pour des applications sur le réseau de distribution électrique [2]. En effet, ce mode d'intégration, basé sur des interactions électriques entre les différentes couches semi-conductrices de la structure, est bien adapté au développement des composants de puissance dont les dispositifs de type triacs et thyristors en sont les premiers exemples. Ces potentialités ont été ensuite augmentées par la pénétration des technologies MOS dans le domaine de la puissance qui a offert un degré de liberté supplémentaire en permettant de supeposer des fonctionnalités en surface de type MOS aux interactions bipolaires en volume.

Sans atteindre la complexité des fonctions obtenues avec les composants Smart Power, les composants réalisés en intégration fonctionnelle intègrent toutefois de nombreuses fonctions de contrôle et de protection autour de l'élément de puissance. Ce mode d'intégration, basé sur des composants de puissance verticaux susceptibles de supporter des tensions de plusieurs centaines de Volts et de transiter des courants de plusieurs Ampères, est mieux adapté au développement de nouvelles fonctions de puissance pour des applications moyenne puissance connectées sur le réseau de distribution de l'énergie électrique. A l'heure actuelle, l'intégration fonctionnelle est en pleine évolution tant sur le plan de la conception de nouvelles fonctions monolithiques de puissance que sur le développement de nouvelles solutions technologiques.

3.2.1 Les associations bipolaires-bipolaires pour des dispositifs plus complexes que le thyristor ou le triac. ST Microelectronics utilise ce concept d'intégration fonctionnelle, dans le développement de nouvelles fonctions de puissance, sous l'appellation ASD ${ }^{\text {тм }}$ (Applications Specific Discretes). L'ASDrm est une approche permettant de répondre rapidement au cahier des charges imposé par un client. La première filière, l'ASD1 тм ou filière Diodes-Bipolaires, est optimisée pour la réalisation de fonctions de protection par réseau de diodes TRANSIL. La seconde, l'ASD2тм ou filiè̀re Bipolaire-Thyristor, repose sur une technologie d'intégration verticale et permet entre autres la réalisation de fonctions basées sur des thyristors, des triacs, des diodes et des transistors bipolaires. Un filière plus récente nommée ASD3 $\square$ ou filière MosThyristors, permet d'introduire les transistors MOS dans la réalisation des structures afin de réaliser des associations MOS-Bipolaires.

L'extension du mode d'intégration fonctionnelle a permis de développer de nouvelles fonctions de commande d'interrupteur et de protection $[5,6,7,8]$, présentant des avantages sur les composants discrets (le gain de place et les performances). La figure 5 représente la coupe d'une structure d'un AC Switch ${ }^{\mathrm{TM}}$ développé par ST Microelectronics Tours [9], et adaptée aux applications sur le réseau électrique. La gâchette, isolée par jonction de la partie de puissance, peut être commandée directement par un circuit micro-contrôleur.
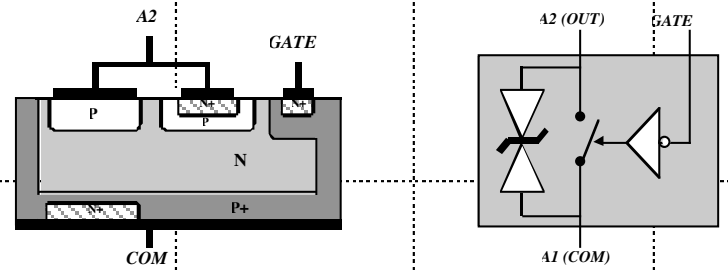

Figure 5. Coupe d'une structure A.C.S. et symbole électrique (structure brevetée).

3.2.2 Évolution vers les associations MOS-bipolaires. Les structures MOS bénéficient d'une entrée à haute impédance (simplifiant considérablement les circuits de commande). Cependant dans des configurations standards de structures MOS verticales adaptées aux applications de puissance, le compromis entre la résistance à l'état passant et la tenue en tension limite leur utilisation dans la gamme des moyenne et basse puissances. Afin de profiter de cette commande en tension, de nombreux travaux ont été développés pour combiner des structures MOS et bipolaires dans les applications "haute tension". L'IGBT, basé sur ce 
type d'association, a connu un essor industriel important ces dernières années. Ce composant réunit l'avantage d'une commande en tension (via une grille de transistor MOS) et d'une faible résistance à l'état passant liée à la modulation de conductivité inhérente à l'injection de portaurs par la région d'anode dans la région $\mathrm{N}^{-}$. Cette approche a ensuite été étendue aux associations MOS-Thyristors qui sont les prémices d'une intégration de nouveaux interupteurs de puissance.

a) Commande des structures MOS-Thyristors à la fermeture. Dans le cas de dispositifs conçus comme les thyristors classiques avec une base large de type $\mathrm{N}$, la commande à la fermeture est réalisée par l'intermédiaire d'un transistor MOS canal $\mathrm{N}$ qui est connecté entre la cathode et la base $\mathrm{N}$ de la structure PNP $[10,11]$. La plupart de ces dispositifs, connus sous l'appellation MOSThyristor ou MOS Gated Thyristors, présentés ces dernières années dans la littérature, dérivent des transistors VDMOS de puissance dans lesquels la région $\mathrm{N}^{+}$de drain est remplacée par une région de type $\mathrm{P}$ fortement dopée afin d'obtenir une structure verticale quatre couches de type thyristor, munie d'une commande isolée (Fig. 6). La résistance de court circuit $R_{c c}$ correspond à la résistance répartie de la région $\mathrm{P}$ sous la cathode $\mathrm{N}^{+}$.

La plupart des composants qui ont été développés dérivent des transistors VDMOS de puissance ou des IGBT et sont de type multicellulaire. Cependant le concept peut s'appliquer à des composants mono-cellulaires conçus à partir de structures thyristors dans lesquelles la région de gâchette est remplacée par une grille MOS [12,13].

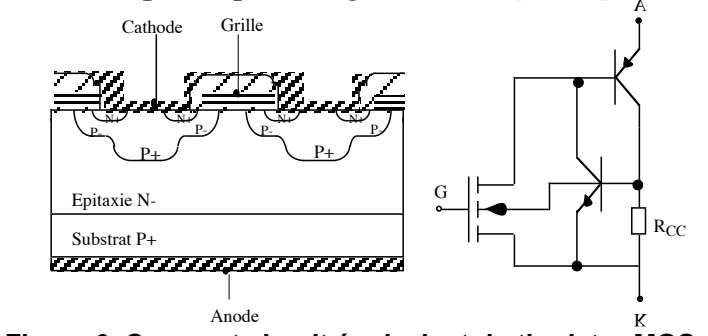

Figure 6. Coupe et circuit équivalent du thyristor MOS.

b) Commande des structures Thyristors MOS à l'ouverture. Plusieurs voies d'intégration ont été envisagées pour réaliser l'ouverture d'une section thyristor par l'intermédiaire d'un transistor MOS [14,15,16,17]. Par exemple, dans le cas du MCT, le transistor MOS, intégré entre la base $\mathrm{P}$ et la cathode, joue le rôle de court-circuit commandable. L'efficacité d'ouverture de ces dispositifs est directement liée aux performances des structures MOS. Les contraintes imposées pour ces structures, en terme d'optimisation du rapport $\mathrm{Z} / \mathrm{L}$, sont similaires à celles rencontrées pour les dispositifs VDMOS basse tension et l'optimisation des caractéristiques électriques passe par une intégration plus poussée et une réduction des dimensions.

3.2.3 Exemples d'in tégration de fonctions spécifiques. Par le passé, la conception de dispositifs de puissance discrets (diodes, transistors bipolaires, MOS, IGBT, thyristors triacs) était effectuée pour une gamme de puissances et de fréquences donnée sans se soucier des caractéristiques précises de l'application future; les électroniciens de puissance en charge des activités "applications" choisissaient dans un catalogue le dispositif le mieux adapté à leur cahier des charges. Cette façon de procéder, séparant de fait les activités des concepteurs de dispositifs et celles des ingénieurs concevant les systèmes de puissance, n'est plus adaptée à la conception de fonctions de puissance intégrées. Cette nouvelle approche consiste à développer des fonctions spécifiques en connaissant a prioni le cahier des charges décrivant la fonctionnalité, les caractéristiques électriques de la fonction, ainsi que les contraintes imposées par son environnement. Ces fonctions spécifiques découlent de fait d'une approche "système" et peuvent être développées en se basant sur le mode d'intégration fonctionnelle. Actudlement, ce concept d'intégration peut être utilisé d'une part pour obtenir des modes de commutation originaux qui ne sont pas exploités dans les composants usuels, et d'autre part pour réaliser de nouvelles fonctions interupteurs réunissant monolithiquement le composant de puissance avec des éléments de protection, d'amplification et de validation de la commande. Cette voie doit à terme conduire à une amélioration des performances, à une meilleure adaptation aux besoins (la conception peut être faite pour une application donnée), et à une plus grande simplicité de conception des équipements.

a) Le thyristor dual. Il a été montré récemment qu'il était possible d'obtenir de nouveaux interupteurs intégrés, tels que le thyristor dual (Fig. 7), en se basant sur le mode d'intégration fonctionnelle [18].La cellule thyristor-MOS autoamorçable et blocable assure l'auto-amorçage du courant $\mathrm{I}_{\mathrm{AK}}$ par passage à zéro de la tension $\mathrm{V}_{\mathrm{AK}}$ ainsi que le blocage de $\mathrm{I}_{\mathrm{AK}}$. La cellule IGBT à canal préformé se comporte comme un générateur de courant et assure le maintien de l'ordre de blocage.

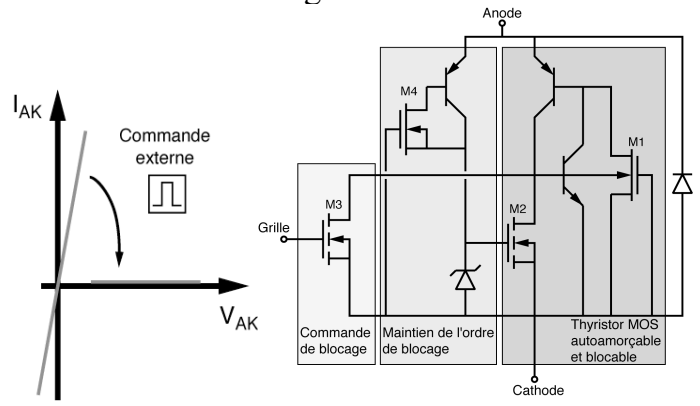

Figure 7. Caractéristique statique du thyristor dual et schéma électrique traduisant cette fonctionnalité.

La cellule NMOS à enrichissement reçoit l'ordre de blocage et la diode PIN assure la bidirectionnalité en courant. Toutefois, ce dispositif possède un courant de fuite à l'état bloqué, lié à l'utilisation d'un transistor NMOS à déplétion pour la commande d'auto-amorçage. Afin de diminuer ce courant de fuite, une nouvelle fonction intégrée "micro-disjoncteur" (Fig. 8a) a été déve-loppée [19]; elle permet de supprimer le courant lié au MOS à canal préformé pour des valeurs de tensions anode-cathode élevées. L'intégration de cette fonction micro-disjoncteur au sein de la structure thyristor dual se traduit uniquement par l'adjonction d'une cellule PMOS à enrichissement (Fig. 8 b).

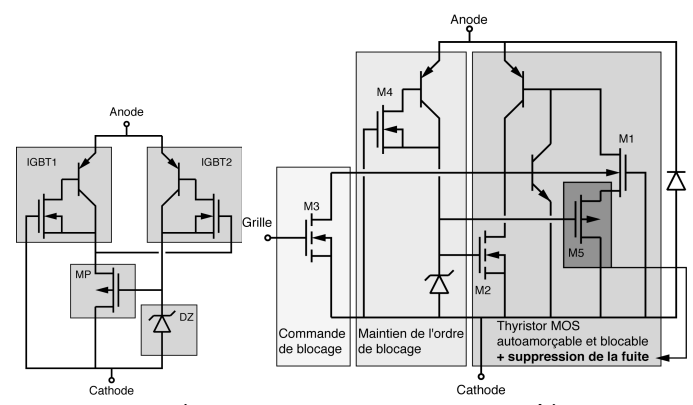

a)

b)

Figure 8. a) Schéma électrique du micro-disjoncteur, b) schéma électrique du thyristor dual complet (sans courant de fuite).

Cet exemple de conception d'une nouvelle fonction de puissance par imbrication de cellules illustre parfaitement l'approche générique du mode d'intégration fonctionnelle. 
b) Interrupteurs bidirectionnels. Actuelement, l'interrupteur bidirectionnel bicommandable (IBB) est synthétisé à partir d'un assemblage de transistors de puissance (MOS, IGBT), de diodes, de circuits intégrés et d'alimentations auxiliaires. Le cœur de cette association est par exemple réalisé par l'association de deux IGBT et de deux diodes. Cette structure pourrait être remplacée avantageusement par deux IGBT symétriques en tension montés en antiparallèle afin de diminuer le nombre de composants et de réduire la chute de tension à l'état passant. La perspective d'intégration totale ou partielle d'un interrupteur bidirectionnel bicommandable passe donc par une première étape relative à l'optimisation d'un IGBT symétrique en tension. ST Microelectronics a développé un dispositif de ce type sous l'appellation MBS (MOS Bidirectionnal Switch). Une périphérie de jonction planar présentant une tenue en tension symétrique (Fig. 9) et compatible avec une filière technologique de type IGBT a été proposée et optimisée récemment au LAAS [20,21].

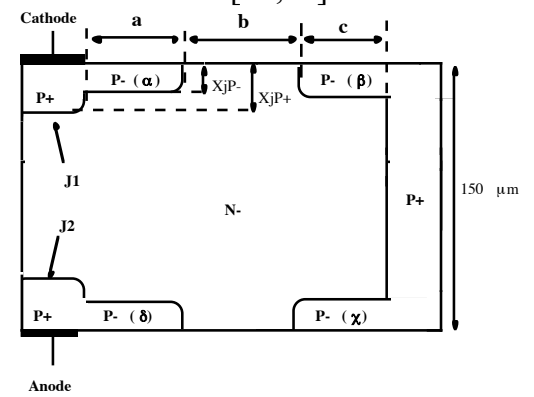

Figure 9. Coupe schématique d'une structure périphérique

Cette périphérie, dont le fonctionnement est basé sur une répartition des équipotentielles dans le volume à travers un caisson $\mathrm{P}$ traversant la plaque de silicium, est constituée de deux terminaisons de type JTE sur chaque face séparées de deux autres régions $\mathrm{P}$ peu dopées reliées par un mur de type $\mathrm{P}$. La réalisation de ces murs $\mathrm{P}^{+}$constitue le point bloquant sur le plan technologique car les diffusions classiques de bore nécessitent des durées importantes, de l'ordre de 200 heures, et peuvent induire des défauts et défomations des plaques. La technique de thermomigration de l'aluminium développée au LAAS [22] et utilisée par ST pour des structures triacs, n'est pas aujourd'hui compatible avec des procédés de fabrication de type IGBT. Ainsi, nous avons proposé une solution technologique permettant la réalisation de ces murs de type $\mathrm{P}$ compatible avec des étapes technologiques permettant de réaliser un IGBT [23]. Il est à noter que ces murs $\mathrm{P}^{+}$ constituent une brique technologique indispensable au développement de nouvelles fonctionnalités [24].

c) Intégration de fonctions de protection. La fiabilité et la disponibilité des systèmes de puissance sont une préoccupation de la recherche actudle en électronique de puissance. Les semi-conducteurs de puissance doivent être performants non seulement en régime normal mais aussi en régime extrême, c'est-à-dire dans des conditions inhabituelles de fonctionnement: surcharge transitoire, régime accidentel tel que le court-circuit, dysfonctionnement du système d'application, fort $\mathrm{di} / \mathrm{dt}$ et $\mathrm{dv} / \mathrm{dt}$, forte énergie dissipée, application spéciale, etc...Ce type de régime entraîne des défaillances de fonctionnement pouvant conduire à la destruction du composant, et éventuellement $\mathrm{du}$ système. L'intégration de circuits de protection spécifiques au cour du composant afin de le protéger des dysfonctionnements du circuit exténeur est donc une contribution appréciable pour l'augmentation de la "fiabilité" et de la disponibilité des systèmes de puissance.

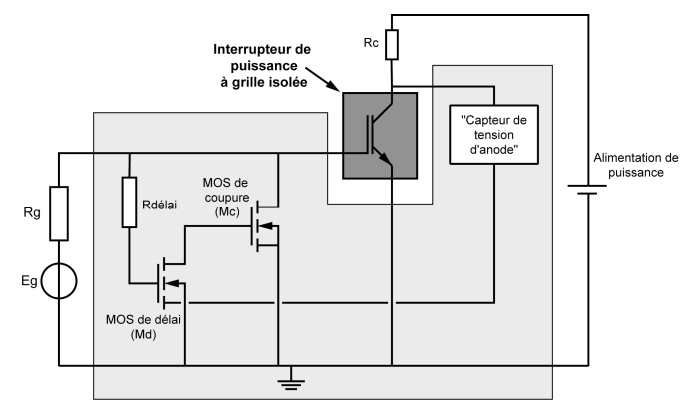

Figure 10. Schéma de principe du circuit de détection et de protection contre les courts-circuits.

Un des points importants concerne la protection des IGBT contre les courts-circuits. Lors de l'apparition d'un court-circuit, l'interrupteur de puissance délivre un courant important avec à ses bornes la pleine tension d'alimentation. Sa destruction est quasi-inévitable en raison du dépassement de la température admissible de jonction. Une solution monolithiquement intégrable a été proposée (Fig. 10) [20].

\subsection{Prolongement de l'in tégration fonctionnelle}

La réduction de la taille et du poids des équipements portables, ainsi que le développement de microsystèmes autonomes sont notamment conditionnés par une optimisation des convertisseurs d'énergie. L'intégration des éléments passifs sur silicium débouchant sur la réalisation de micro-condensateurs sur silicium constitue un élément de réponse à cette évolution. Ces niches stratégiques ont conduit au développement de travaux relatifs à la réalisation d'éléments passifs sur silicium dans la perspective d'évoluer vers des convertisseurs monolithiques. Cette approche doit permettre de diminuer le nombre de composants et les connexions en vue de conduire à des produits plus fiables et moins onéreux. De nombreuses applications de filtrage basées sur de réseaux RL sont également concernées. Les récents développements dans le domaine des microtechnologies sont bien adaptés à la réalisation de bobines sur silicium. La réalisation de moules de résines et l'électrochimie de matériaux conducteurs ou magnétiques permettent de réaliser des noyaux magnétiques et des enroulements sur des épaisseurs de plusieurs dizaines de microns. Généralement les procédés de technologie dite froide permettent de faire du "post processing" et donc d'envisager une intégration avec les dispositifs semiconducteurs. Des premiers prototypes présentant une valeur d'inductance de $35 \mathrm{nH} / \mathrm{mm}^{2}$ à $1 \mathrm{Mhz}$ ont été réalisés. Le procédé de fabrication utilisé est entiòrement compatible avec un procédé CMOS standard, et des microconvertisseurs présentant une puissance de sortie de 1 à 2 $W$ sont envisageables $[25,26]$. La réalisation de condensateurs sur silicium peut s'adresser d'une part aux applications de filtrage sous basse tension (quelques Volts pour les micro-convertisseurs) avec des condensateurs à capacité constante, et d'autre part, pour des tensions plus élevées (quelques centaines de Volts), à des circuits d'aide à la commutation à l'aide de condensateurs à capacités non linéaires permettant d'améliorer les conditions de commutation des interrupteurs de puissance. Leur fabrication est basée sur la technique de gravure profonde [27]. En effet, la topologie dite "en tranchées", permet d'augmenter le rapport entre la surface effective du condensateur et la surface de la puce. La valeur de la capacité est ensuite liée aux caractéristiques de l'isolant et peut être augmentée en utilisant des diélectriques à forte permittivité déposés en couche mince. L'association de structures MIS (Métal-Isolant-Semi-conducteur) et de 
jonctions $\mathrm{PN}^{-}$permet d'obtenir la non-linéarité. Le principe de ces dispositifs repose sur le contrôle de l'accès à une capacité constante (capacité MIS) grâce à l'extension de la zone de charge d'espace de la jonction $\mathrm{PN}^{-}$.

\section{Conclusion}

Suivant les niveaux de courant et de tension traités, les solutions adoptées pour l'intégration en électronique de puissance sont donc basées sur une intégration monolithique ou hybride.

L'intégration hybride a permis d'exploiter au mieux les performances des nouveaux composants de puissance et de les destiner à des applications moyenne et forte puissances. Les principaux travaux se sont focalisés sur le "packaging", et plus particulièrement sur les matériaux isolants, la connectique, le refroidissement et les interfaces entre les différents maténiaux.

L'intégration monolithique a été favorisée dans un premier temps par l'évolution des technologies dédiées aux circuits intégrés de traitement du signal et de l'information. Les stratégies d'intégration ont suivi deux voies: les circuits Smart Power et le mode d'intégration fonctionnelle. Dans le premier cas, l'intégration peut être vue comme un prolongement de l'intégration du traitement du signal en rajoutant un étage de puissance à un circuit intégré classique. Les problématiques étudiées étaient essentiellement centrées sur les techniques d'isolation entre la partie puissance et la partie basse tension. Pour des puissances plus élevées, l'intégration fonctionnelle est basée sur l'utilisation d'interactions électriques au sein du semiconducteur afin de proposer de nouvelles fonctions pour le traitement de l'énergie électrique. D'abord centrées sur des associations bipolaires-bipolaires, qui ont permis le développement des filières ASD de ST Microelectronics, l'introduction de technologies MOS a permis d'augmenter la fonctionnalité en associant des effets MOS en surface aux interactions bipolaires dans le volume.

Le premier constat que nous pouvons faire est que pour la conversion de l'énergie, l'intégration n'est pas, et ne peut être considérée comme une simple miniaturisation, mais comme l'apport de solutions innovantes pour assurer des fonctionnalités bien identifiées. L'intégration doit être basée sur une démarche "système" à partir des besoins identifiés au niveau des applications et nécessite maintenant des développements technologiques spécifiques tant au niveau des matériaux que des procédés d'élaboration ainsi que des modèles et des outils adaptés pour la conception.

Dans de nombreux domaines (télécommunications, électronique grand public, domotique, informatique, transports...), cette évolution doit se faire inévitablement vers l'intégration totale basée le plus souvent sur une fabrication collective afin de procurer une avancée identique à celle connue aujourd'hui dans le domaine du traitement du signal. En fonction des niveaux de puissance et des applications envisagées, cette intégration sera, soit monolithique, soit hybride, mais dans tous les cas hétérogène du fait de la diversité des éléments à intégrer (éléments actifs, passifs, refroidisseurs, connectique). Dans cette perspective, l'élément de base ne sera plus le semiconducteur de puissance associé à d'autres dispositifs (alimentations, éléments passifs, refroidisseurs) mais la cellule de commutation. Dans ce nouveau contexte la conception et le mode de réalisation des systèmes de puissance doit se faire dès le départ en prenant en compte le système complet, ainsi que les possibilités d'intégration offertes par les nouvelles technologies. Il faut toutefois noter que cette intégration n'est pas une fin en soi et doit répondre à une augmentation des performances, notamment du rendement, de la sécurité, et de la fiabilité des fonctions de conversion de l'énergie électrique tout en diminuant l'encombrement, les coûts et les problèmes de CEM.

\section{Références}

[1] E.R. Motto, "Application Specific Intelligent Power Modules - A novel Approach to System Integration in Low Power Drives", www.pwrx.com.

[2] J-L. Sanchez, "State of the art and trends in power integration", MSM, Puerto Rico(USA), 1999, p. 20-29.

[3] G. Charitat, J-L. Sanchez, P. Rossel, H. Tranduc, M. Bafleur, "Power Integrations : Overview and Future", Mix VLSI'95, Mixed Design of VLSI circuits, Cracovie (Pologne), 1995, p. 47-59.

[4] V. Rumennik, "Power devices are in the chip", IEEE Spectrum, 1985, p. 42-48.

[5] R. Pezzani, "Monolithic semiconductor switch and supply circuit component", Patent Number : 5883 401, 1996.

[6] R. Pezzani, "Three-state monolithic static switch", Patent Number : 5 $883500,1997$.

[7] R. Pezzani and E. Bernier, "Programmable protection circuit and its monolithic manufacturing", Patent Number : RE 35 854, 1995

[8] R. Pezzani, "Thyristor control switch for a bidirectional motor", Patent Number : 5889 374, 1997.

[9] L. Gonthier, J. Mathias, F. Duclos, "A new Overvoltage-Protected Logic Level AC Switch Thanks to Functional Integration", EPE'99, Lausanne, Suisse.

[10] B.J. Baliga, "Enhancement - and depletion - mode vertical - channel MOS gated thyristors", Electronics Letters Vol. 15, n²0, 1979.

[11]A. Jaecklin, "A N FET-Drivin power thyristor", IEEE Transactions on Electron. Devices, Vol. ED-34, n5, 1987 .

[12] D. Darees, "Contribution à l'étude d'associations monolithiques de composants MOS et bipolaires : le thyristor à gachette isolée", Thèse de Docteur Ingénieur INSA Toulouse, Février 1986.

[13] J-L. Sanchez et P. Leturcq, "Thyristor à gachette isolée planar haute tension (1400 Volts): un exemple d'interrupteur intégré de puissance", EPF90, Toulouse, 1990.

[14] B.J. Baliga, "The MOS-gated emitter switched thyristor", IEEE Electron Device Lett, Vol.EDL-11, 1990,p. 75-77.

[15] K. Lilja and T. Stockmeier, "The FIBS, a new high voltage BiMOS switch", ISPSD'92, Tokyo, 1992, p. 261-265.

[16] V.A.K. Temple, "MOS controlled thyristor - A new class of power devices", IEEE Transactions on Electron. Devices, Vol. 33, $n^{\circ} 10$, 1986.

[17] F. Bauer, "Design aspects of MOS-Controlled thyristor elements : Technology, simulation and experimental results", IEEE Transactions on Electron. Devices, Vol. 38, n7, 1991.

[18] M. Breil "Etude d'associations MOS-Thyristor autoamorçables et blocables. Exemple d'intégration de la fonction thyristor dual", Thèse de doctorat de l'Institut National des Sciences Appliquées, Toulouse, Janvier 1998.

[19] J.P. Laur, J.L. Sanchez, M. Marmouget, P. Austin, J. Jalade, M. Breil and M. Roy, "A new circuit-breaker integrated device for protection applications", ISPSD99, Toronto, 1999, p. 315-318.

[20] O. Causse, "Contribution à l'amélioration de la fonctionalité et des performances de l'IGBT", Thèse de doctorat de l'Université PaulSabatier, Toulouse, Juin 2001.

[21] O. Causse, P. Austin, J.L. Sanchez, G. Bonnet and E. Scheid, "Achievement of a new peripheral planar structure supporting a symmetrical blocking voltage", EPE'01, Graz, 2001.

[22] J.M. Dilhac, L. Cornibert, B. Morillon, S. Roux and C. Ganibal, "Industrial relevance of deep junctions produced by rapid thermal processing for power integrated devices", ISPSD'99, Toronto, 1999, p. 237-240.

[23] J-L. Sanchez, E. Scheid, P. Austin, M. Breil H. Carrière, P. Dubreuil, E. Imbernon, F. Rossel, B. Rousset, "Realization of vertical $\mathrm{P}+$ walls through wafer for bidirectional current and voltage power integrated devices", ISPSD'03, à paraître, Cambridge, Angleterre.

[24] A. Bourennane, M. Breil, J-L. Sanchez, P. Austin, J. Jalade, "New triggering mode in a vertical bidirectional MOS-thyristor device", ISPS02, Prague, 2002,p. 101-106, 2002.

[25] S. Sugahhara, M. Edo, T. Sato and K. Yamasawa, "The optimum chip size of a thin film reactor for a high-efficiency operation of a micro DC-DC converter", PESC'98, p. 1499-1503.

[26] C.R. Sullivan and S.R. Sanders, "Measured performance of a HighPower Density Microfabricated transformer in a DC-DC Converter", PESC'96.

[27] H. Hakim "Intégration de composants passifs sur silicium", Thèse de doctorat de l'Institut National des Sciences Appliquées, Toulouse, Juillet 2001 